\title{
PPARG2 Pro12Ala polymorphism influences body composition changes in severely obese patients consuming extra virgin olive oil: a randomized clinical trial
}

\author{
Ana Paula Santos Rodrigues ${ }^{*}$ (D), Lorena Pereira Souza Rosa and Erika Aparecida Silveira
}

\begin{abstract}
Background: Previous intervention studies have reported the association of the PPARG2 Pro12Ala (rs1801282) and IL6 -174G > C (rs1800795) polymorphisms with weight loss; however, their results are inconsistent. We aimed to investigate the effect of the PPARG2 Pro12Ala and IL6 -174G >C polymorphisms on body weight, body composition and metabolic parameters after a 12-week nutritional intervention with a traditional Brazilian diet and extra virgin olive oil supplementation in severely obese patients.

Methods: A total of 149 severely obese individuals [body mass index $(\mathrm{BMI}) \geq 35 \mathrm{~kg} / \mathrm{m}^{2}$ ] were randomized into three 12-week nutritional intervention groups - the extra virgin olive oil supplementation (OO) group $(n=50)$, the traditional Brazilian diet (DieTBra) group $(n=49)$, and the DieTBra plus extra virgin olive oil supplementation (DieTBra+OO) group ( $n=50)$. Anthropometric measurements, body composition, metabolic parameters, physical activity practise and dietary intake were assessed. The associations were tested using generalized linear models adjusted for confounders.
\end{abstract}

Results: The PPARG2 Pro12Ala polymorphism influenced body composition changes. Ala carriers in the intervention groups with extra virgin olive oil supplementation had greater reductions in the percentage of body fat (\%BF) (OO: $p=0.049$, DietBra+OO: $p=0.004$ ) and greater increases in both fat-free mass (FFM) (OO: $p=0.020$, DieTBra: $p=0$. $007)$ and lean mass $(L M)(O O: p=0.020$, DieTBra+OO: $p=0.007)$ than did ProPro homozygotes. No association was found for the $1 / 6-174 G>C$ polymorphism.

Conclusions: Extra virgin olive oil intake may modulate favourable body composition changes, promoting a decrease in the \%BF and increases in the LM and FFM of severely obese individuals, even without weight loss, in the presence of the Ala allele of the Pro12Ala polymorphism.

Trial registration: Registered under ClinicalTrials.gov Identifier No. NCT02463435.

Keywords: PPAR gamma, Interleukin-6, Weight loss, Diet, Body composition, Adiposity

\footnotetext{
* Correspondence: anapsr@gmail.com

Programa de Pós-Graduação em Ciências da Saúde, Faculdade de Medicina,

Universidade Federal de Goiás, 1a Avenida, s/n, Setor Leste Universitário,

Goiânia, Goiás CEP 74605-020, Brazil
}

(c) The Author(s). 2018 Open Access This article is distributed under the terms of the Creative Commons Attribution 4.0 International License (http://creativecommons.org/licenses/by/4.0/), which permits unrestricted use, distribution, and reproduction in any medium, provided you give appropriate credit to the original author(s) and the source, provide a link to the Creative Commons license, and indicate if changes were made. The Creative Commons Public Domain Dedication waiver (http://creativecommons.org/publicdomain/zero/1.0/) applies to the data made available in this article, unless otherwise stated. 


\section{Background}

An obesogenic environment has an overriding importance in the development of obesity; however, genetic susceptibility also plays a critical role [1]. Different responses to weight loss interventions among individuals may be related to genetic variations [2, 3]. In this sense, many polymorphisms of genes involved in adipogenesis, lipid metabolism, energy expenditure, and the regulation of appetite and food intake have been studied [4, 5]. The Pro12Ala (rs1801282) polymorphism, which is the most studied polymorphism in the peroxisome proliferator-activated receptor $\gamma$ gene (PPARG2), and the $-174 \mathrm{G}>\mathrm{C}$ (rs1800795) polymorphism in the promoter region of the interleukin-6 gene (IL6) have been reported to influence weight loss treatment outcomes [2,6].

The PPARG2 gene controls the expression of genes involved in adipocyte differentiation, fatty acid glucose metabolism, and inflammatory processes [7]. The Ala allele of the Pro12Ala polymorphism has been associated with elevated BMI, especially among severely obese individuals [8]. Increasing amounts of data on the Pro12Ala polymorphism have shown that gene-diet interactions are responsible for the weight loss variability in intervention studies, partly in conjunction with fatty acid intake [9].

The $-174 \mathrm{G}>\mathrm{C}$ polymorphism (rs1800795) has been shown to influence the transcriptional regulation and plasma levels of the pro-inflammatory cytokine IL- $6[10$, 11]. However, the association between the $C$ allele and increased BMI is inconsistent [12, 13]. Intervention studies have reported the association of the $\mathrm{C}$ allele with greater weight reduction in individuals with high cardiovascular risk [14], protection against weight regain in obese individuals, and improvement in the ability to maintain weight in the presence of the Pro12Ala Ala allele [15]. Nevertheless, the $\mathrm{C}$ allele also induced resistance to weight loss in morbidly obese individuals after laparoscopic gastric banding [16].

Recently, weight loss intervention studies have focused on healthy eating patterns, such as the Mediterranean Diet (MedDiet). In addition to cardiometabolic benefits, MedDiet has also been associated with weight loss and lower BMI, and olive oil is one of the most important components of this eating pattern [17]. In Brazil, the traditional eating pattern, which is based on the consumption of rice and beans, is also considered healthy and is associated with lower levels of overweight and obesity [18-20]. The consumption of olive oil has been increasing not only in Brazil but also in other countries [21], and the effects of olive oil on other dietary patterns should be investigated.

Given the controversial results about the impact of the Pro12Ala and $-174 \mathrm{G}>\mathrm{C}$ variants on the weight loss response to lifestyle interventions and the need for more effective nutritional strategies to fight severe obesity, randomized intervention trials may provide the strongest evidence in this matter. Thus, the aim of the current study was to assess the effect of the PPARG2 Pro12Ala and IL6 $-174 \mathrm{G}>\mathrm{C}$ polymorphisms on the body weight, body composition and metabolic parameters of severely obese patients after a 12-week nutritional intervention with a traditional Brazilian diet and extra virgin olive oil supplementation. Moreover, the association of the aforementioned polymorphisms with diet-induced weight loss has been poorly investigated in severely obese individuals.

\section{Methods}

\section{Study design and subjects}

Severely obese patients (BMI $\geq 35 \mathrm{~kg} / \mathrm{m}^{2}$ ) were investigated according to a parallel group, single-blinded, controlled randomized clinical trial with nutritional intervention and extra virgin olive oil supplementation (DieTBra Trial) for 12 weeks. The study was conducted from June 2015 to February 2016 in Goiânia, Goiás State, Brazil, at the Nutrition in Severe Obesity Outpatient Clinic of the Clinical Research Unit of the Clinical Hospital/Federal University of Goiás.

All participants provided written informed consent prior to enrolment. The study protocols were performed in accordance with the ethical standards specified in the 1961 Declaration of Helsinki (as revised in Hong Kong in 1989, in Edinburgh in 2000, and in South Korea in 2008) and were approved by the Ethics Committee on Research with Humans of the Clinical Hospital/Federal University of Goiás under protocol number 747.792. The trial was also registered at ClinicalTrials.gov (NCT02463435).

Our study included patients with a BMI $\geq 35 \mathrm{~kg} / \mathrm{m}^{2}$ and an age between 18 and 65 years who were referred to our outpatient clinic from the primary care of the Brazilian Unified Health System (SUS) in Goiânia. SUS is a public health care system designed to offer free and universal health coverage in Brazil and is divided into basic, specialized and high complexity categories of assistance. We excluded volunteers who had already undergone bariatric surgery; had been under nutritional treatment for weight loss within the previous 2 years; were currently taking anti-obesity or anti-inflammatory drugs; had experienced a weight loss higher than $8 \%$ in the last 3 months; had been diagnosed with HIV/AIDS or had heart/kidney/hepatic insufficiency, chronic obstructive pulmonary disease, or cancer; or were currently pregnant.

\section{Baseline, randomization, and blinding}

After enrolment, patients underwent a baseline phase that was divided into two parts due to the large amount 
of data to be collected and to allow the assessment of physical activity by an accelerometer for seven consecutive days. In baseline part 1, we collected sociodemographic, clinical, and anthropometrical data. At that time, the accelerometer was also fastened onto the patients' wrist. In baseline part 2, body composition and dietary intake were assessed, blood samples were drawn from each participant, and the accelerometer was retrieved. At the end of the baseline phase, the study participants were randomly assigned by the same trained researcher into three intervention groups in 1:1:1 ratio using an algorithm available at www.randomization.com.

Randomization resulted in the placement of the participants into three groups, namely: 1) the olive oil supplementation (OO) group, which received extra virgin olive oil supplementation of $52 \mathrm{~mL} / \mathrm{d}, 2$ ) the traditional Brazilian diet (DieTBra) group, which received nutritional intervention for weight loss, and 3) the traditional Brazilian diet plus olive oil supplementation (DieT$\mathrm{Bra}+\mathrm{OO})$ group, which received the same intervention as the DieTBra group in addition to extra virgin olive oil supplementation of $52 \mathrm{~mL} / \mathrm{d}$. Despite the difficulties of and barriers to blinding dietary interventions in clinical trials [22], in this study, patients were blinded to the type of supplement consumed. To assure blinding, patients in the $\mathrm{OO}$ and DieTBra+OO groups were told that the sachets of extra virgin olive oil were a dietary supplement enriched with bioactive compounds in an oily form. The information on the supplement sachets was adequately prepared according to the recommendations of the National Health Surveillance Agency of Brazil for Clinical Trials to mask this intervention. The research team was trained to use the term "dietary supplement enriched with bioactive compounds" or just "nutritional supplement" to describe the olive oil to the patients in the groups that received the supplement. Furthermore, the follow-up visits of each intervention group occurred on a different day of the week to avoid contact among the participants. Fig. 1 shows the flow chart of the participants in the study.

\section{Interventions and follow-up visits}

The DieTBra nutritional intervention comprises a restricted energy food plan for weight loss. The formulation of the food plan was based on the traditional Brazilian diet, which consisted of rescuing the healthy Brazilian eating habits used by the general population in Brazil before the nutritional transition occurred. The nutritional transition changed the food pattern by including fast food and large amount of ultra-processed foods in the diet. DieTBra includes rice and beans, along with a small portion of meat or poultry, and fresh raw and cooked vegetables in the main meals. Fruits are common at breakfast and at other smaller meals; dairy products, bread and eggs are also common [23, 24]. Furthermore, the recommendations of the new Food Guide for the Brazilian Population were applied. All patients were encouraged to eat fresh and/or minimally processed foods instead of ultra-processed foods [24]. An individualized and balanced food plan was prescribed; the plan was divided in four to six meals a day and considered cultural, social, and economic reality as well as aspects of eating behaviour [24].

We used a specific equation for severely obese patients to calculate resting energy expenditure [25]. Total energy expenditure was determined using physical activity level factors [26], and the thermic effect of food [27]. Total food plan energy was determined based on a weight reduction goal for the 12-week follow-up according to the patient's BMI (5 to $10 \%$ weight loss), resulting in a daily restriction of 550 to $1100 \mathrm{kcal}$. Macronutrient distribution followed the Dietary Reference Intake (DRI) recommendations: $45-65 \%$ carbohydrates, $10-35 \%$ proteins, and $20-35 \%$ lipids [26].

Additionally, groups prescribed DieTBra also received nutritional instructions according to metabolic disturbances and comorbidities and instructions about physical activity practice according to the World Health Organization's recommendation (at least $150 \mathrm{~min}$ of moderate-intensity aerobic physical activity per week) [28].

Patients in the OO group received only the extra virgin olive oil supplementation $(52 \mathrm{~mL} / \mathrm{d}$ divided into 4 sachets of $13 \mathrm{~mL}$ each) and were instructed to maintain their usual diet. The DieTBra+OO group received the nutritional intervention as above plus extra virgin olive oil supplementation $(52 \mathrm{~mL} / \mathrm{d}$ in 4 sachets of $13 \mathrm{~mL}$ each). The total food plan energy for the DieTBra+OO patients was adjusted to include the extra virgin olive oil supplement.

At the end of each visit, patients were provided, at no cost, a sufficient quantity of extra virgin olive oil sachets to last until the next visit (4 weeks). Patients were instructed about the consumption of the extra virgin olive oil (preferably at lunch and dinner, not to be used for cooking, and not to be shared with others). To control the consumption of the extra virgin olive oil, patients were asked to return all sachets, even the ones not consumed. The intervention lasted 12 weeks, and visits took place every 4 weeks. Trained registered dietitians delivered the interventions. Standard operating procedures were developed to train the entire research team on the measurements and assessments performed throughout the study, and periodic training and group meetings were conducted to assure the quality of the data collected.

\section{Body composition measurements}

The anthropometric measurements included height and body weight. Measurements were performed using 


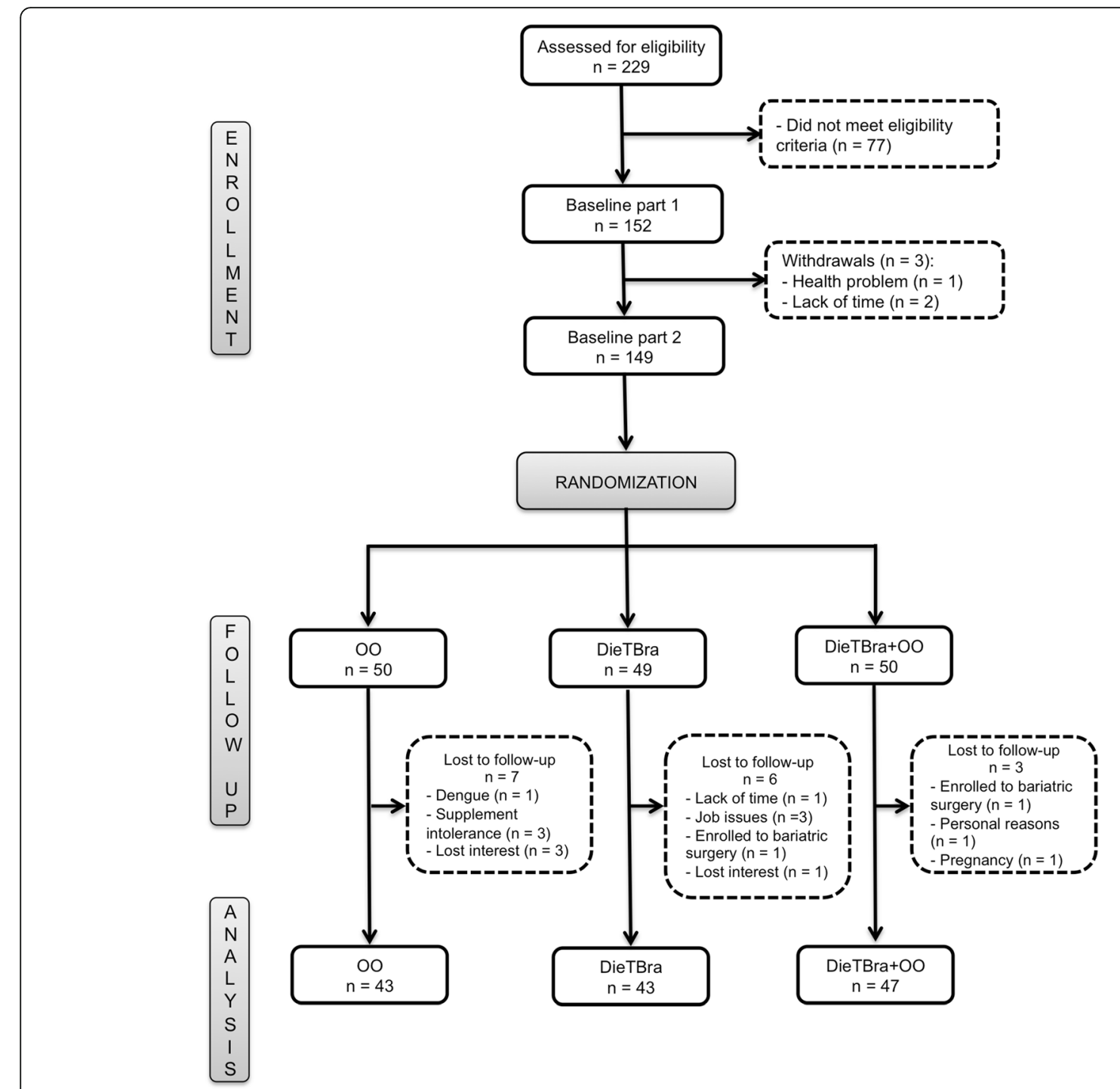

Fig. 1 Flow chart of the study participants

standardized procedures [29]. BMI was calculated by dividing the body mass $(\mathrm{kg})$ by the square of the height $\left(\mathrm{m}^{2}\right)$. Severe obesity was defined as a BMI $\geq 35 \mathrm{~kg} / \mathrm{m}^{2}$ [30].

Fat mass $(\mathrm{kg})$, fat-free mass $(\mathrm{kg})$, percentage of body fat $(\%)$ and lean mass $(\mathrm{kg})$ were assessed by dual-energy X-ray absorptiometry (DXA) using a Lunar DPX NT device (GE Healthcare, Madison, WI, USA). The measurement criteria were as follows: a 12-h fast prior to measurement, the avoidance of strenuous physical activity and alcohol, as well as food and drinks containing caffeine, on the day prior to measurement, and the absence of pacemakers and orthopaedic prostheses/implants (devices containing metal). DXA assessment was performed on patients lying in a supine position on the scanner table with arms close to the body. Duplication of right arm data using the hemiscan technique was performed when the subject was too large for the equipment to capture both arms [31]. DXA assessment was limited to patients with a body weight up to $130 \mathrm{~kg}$ due to the device capacity. Body weight and DXA measurements were performed at baseline part 2 and at week 12 .

\section{Dietary intake}

Twenty-four-hour records were used to assess dietary intake in the baseline phase and at final follow-up. Trained registered dietitians collected three 24-h records within 7 days using the Multiple-Pass Method; two were collected face-to-face and one was collected over the phone [32]. A nutritional analysis was performed using Avanutri Online (Avanutri Equipamentos de Avaliação Ltda, Rio de Janeiro, RJ, BR). Energy (kcal), proteins (\%), carbohydrates (\%), lipids (\%), saturated fatty acids (SAF) (\%), monounsaturated fatty acids (MUFA) (\%), 
polyunsaturated fatty acids (PUFA) (\%), and the polyunsaturated:saturated fatty acids ratio (P:S ratio) were obtained by calculating the means of these parameters from the three $24-\mathrm{h}$ records.

\section{Physical activity assessment}

We measured physical activity objectively using the tri-axial accelerometer ActiGraph wGT3X (ActiGraph, Pensacola, FL, USA). At baseline and at final follow-up, study participants were instructed to wear the accelerometer on the non-dominant wrist $24 \mathrm{~h}$ a day, even when showering and at bedtime, for seven consecutive days. The device was programmed by the researcher to capture data from midnight starting the day after the allotment until midnight ending the day before the collection, thus capturing six full days of data. The wGT3X measured the acceleration in three axes $(x, y, z)$ within a dynamic range of $\pm 8 \mathrm{~g}$ and a sampling frequency of $30 \mathrm{~Hz}$.

We used ActiLife 6.11.7 software to initialize and download the accelerometer data. Individuals were included in the analysis if data were available for at least $50 \%$ of the wear time. Output data were processed using the $\mathrm{R}$ package GGIR (http://cran.r-project.org). Vector magnitude of activity-related acceleration (three axes) was calculated using the Euclidian norm minus $1 \mathrm{~g}$ (ENMO: $\sqrt{ } \mathrm{x}^{2}+\mathrm{y}^{2}+$ $\left.\mathrm{z}^{2}-1 \mathrm{~g}\right)$. Activity intensity was estimated as the average time per day spent in moderate and vigorous physical activity from 5-s aggregated time series. The outcomes used in the present study were moderate-to-vigorous physical activity (MVPA), which was considered to include activities with an acceleration equal to or greater than $100 \mathrm{mg}$ [33] with an estimated time spent equal to or greater than 10 min per bout during a week; and sedentary time, which was considered to include activities with an acceleration lower than $50 \mathrm{mg}$ (non-bouted) per day. Bouts of MVPA were defined as 10-min time windows that started with a 5-s epoch value equal to or greater than $100 \mathrm{mg}$ and for which $80 \%$ of the subsequent 5 -s epoch values were equal to or greater than the $100 \mathrm{mg}$ threshold [34].

\section{Biochemical analysis}

Blood samples for biochemical analysis and genotyping were collected after 12-h overnight fasting at baseline and at week 12 . Serum glucose, total cholesterol, high-density lipoprotein (HDL), low-density lipoprotein (LDL), and triglycerides were measured by enzyme-colorimetric methods. Serum insulin was measured by chemiluminescence, and haemoglobin A1c (HbA1c) was measured by liquid chromatography. The homeostasis model assessment of insulin resistance (HOMA-IR) was calculated following the formula of Matthews et al. [35].

\section{DNA extraction and genotyping}

DNA was extracted from whole blood using a commercial kit (PureLink ${ }^{\mathrm{Tm}}$ Genomic DNA Mini Kit; Invitrogen, Carlsbad, CA, USA). DNA concentration and purity were evaluated by spectrophotometric determination of the $A_{260 / 280}$ ratio using a NanoDrop 2000c (Thermo Fisher Scientific, Waltham, MA, USA). DNA quality was assessed using agarose gel electrophoresis. Genotyping of PPARG2 (Pro12Ala, rs1801282) and IL6 (-174G > C, rs1800795) was carried out using a TaqMan ${ }^{\circ}$ SNP Genotyping assay (Applied Biosystems, Foster City, CA, USA) for each polymorphism with a StepOne Real-Time PCR system (Thermo Fisher Scientific, Waltham, MA, USA). For quantitative polymerase chain reaction (qPCR), a TaqMan GTExpress ${ }^{\text {Th }}$ Master Mix (Thermo Fisher Scientific, Waltham, MA, USA) reagent kit was used, and qPCR was performed in a final volume of $21 \mu \mathrm{L}$ according to the manufacturer's instructions. Although DNA samples were extracted for all study participants, the qPCR amplification was only conducted for the PPARG2 Pro12Ala polymorphism on samples from 145 individuals and for the IL6 -174G > C polymorphism on samples from 147 individuals.

\section{Statistical analysis}

We structured the dataset in EpiData 3.1 with double entry typing and validation for the assessment of consistency. Data are expressed as the means \pm SD for continuous variables and as frequencies and percentages for categorical variables. The Kolmogorov-Smirnov test was used to analyse the distribution of continuous data. The homogeneity among the nutritional intervention groups was tested using analysis of variance (ANOVA) or a Kruskal-Wallis test and a Chi-squared test. The Hardy-Weinberg equilibrium was tested in the population using a Chi-squared test. The mean daily extra virgin olive oil intake was compared between the $\mathrm{OO}$ and DieTBra+OO groups using Student's t-test. The delta (12th week measurement - baseline measurement) was calculated for food intake variables. Comparison of food intake means was performed using a Wilcoxon signed-rank test and a Kruskal-Wallis test followed by post hoc Bonferroni correction.

We investigated the changes in the anthropometric and body composition measurements as primary outcomes and the changes in the metabolic markers as secondary outcomes. General linear models (GLMs) were performed to test the main effects of the polymorphisms and nutritional interventions on the outcomes. Models were adjusted for potential confounders such as age, sex, baseline BMI and sedentary time. Multiplicative interaction terms (polymorphism and sex-by-nutritional intervention) were used in the models to test the statistical homogeneity of the effects, and as there was no 
interaction, we examined the main effects after removing the interaction term from the model. When effects were found for a polymorphism, we compared the outcome by genotypes (dominant model) for each nutritional intervention using Student's t-test.

Data analyses were performed using STATA version 12.0 (StataCorp, College Station, TX, USA), and statistical significance was defined at $P<0.05$.

\section{Results}

Baseline characteristics of the studied participants are presented in Table 1. As expected, due to randomization, there were no significant differences between the nutritional intervention groups at baseline. The genotype distribution of both polymorphisms did not deviate from the Hardy-Weinberg equilibrium expectation $(p=0.689$ for PPARG2 Pro12Ala, and $p=0.863$ for IL6 $-174 \mathrm{G}>\mathrm{C}$ ). All analyses were conducted with a dominant genetic model because of the small number of AlaAla homozygotes for the Pro12Ala polymorphism and CC homozygotes for the $-174 \mathrm{G}>\mathrm{C}$ polymorphism. The retention rate in the current study was $89 \%$. Olive oil consumption was similar $(p=0.731)$ in $\mathrm{OO}$ and DieTBra+OO (40.6 \pm 11.1 and $39.8 \pm 10.7$, respectively).

Interventions promoted significant changes in the food intake of the study participants. At the end of follow-up, the DieTBra and DieTBra+OO groups had a decreased total energy intake compared to that at baseline. The delta for the total energy intake showed a greater reduction in both of those groups than in the OO group. Considering total fat intake, the DieTBra group had a reduced consumption, while an increased consumption was observed in the $\mathrm{OO}$ and DieTBra+OO groups between baseline and final follow-up. The delta for total fat intake was greater in the $\mathrm{OO}$ and DieTBra+OO groups than in the DieTBra group. Regarding carbohydrates, PUFA and SAF, we observed significant reductions in the $\mathrm{OO}$ and DieTBra+OO groups between baseline and final follow-up, but the delta for these variables was not different between the intervention groups (Table 2).

Analyses of the effects of the polymorphisms and nutritional interventions on anthropometric measurements, body composition and metabolic markers were performed using GLMs. Tables 3 and 4 show the GLMs for the outcomes that presented significant effects. We found an effect of the Pro12Ala polymorphism on the percentage of body fat (\%BF), fat-free mass (FFM) and lean mass (LM) (Table 3). Additional comparison between genotypes showed that Ala carriers in the $\mathrm{OO}$ and DieTBra+OO groups had a greater reduction in \%BF than did ProPro homozygotes. FFM and LM showed significant increases for Ala carriers from the $\mathrm{OO}$ and DieTBra+OO groups compared to those for ProPro homozygotes. No differences were found between genotypes in the DieTBra group (Fig. 2).

DieTBra and DieTBra+OO promoted changes in weight and body composition that were independent of the polymorphisms. Considering the models for the Pro12Ala polymorphism, the weight reduction was greater for DieTBra and DieTBra+OO than for OO (Table 3). Considering the models for $-174 \mathrm{G}>\mathrm{C}$ polymorphism, the weight reduction was greater for DieTBra and DieTBra+OO than for $\mathrm{OO}$, but the results were not independent of sex. Additionally, fat mass showed a greater reduction for DieTBra+OO than for OO (Table 4).

The nutritional interventions and the polymorphisms did not affect metabolic parameters. Data for the changes in all body composition and metabolic parameters according to the dietary intervention are shown in the supplemental material (Additional file 1: Table S1).

\section{Discussion}

The key finding of the current study is the significant association between the Pro12Ala (rs1801282) polymorphism and body composition changes in severely obese patients after a 12-week intervention with extra virgin olive oil. Ala carriers that consumed extra virgin olive oil with or without DieTBra had a greater reduction in \%BF and had a greater increase in FFM and LM than did ProPro homozygotes, while DieTBra had the same effect on ProPro homozygotes as it did on Ala carriers. To our knowledge, this is the first study demonstrating a synergistic effect of extra virgin olive oil intake and the Pro12Ala polymorphism on \%BF, FFM and LM. These findings contribute to expand the knowledge about gene-diet interactions, showing that extra virgin olive oil may modulate favourable changes in body composition in the presence of the Ala allele of the PPARG2 Pro12Ala polymorphism.

In our study, Ala carriers had greater reduction in \%BF and a greater increase in FFM and LM than ProPro homozygotes when consuming extra virgin olive oil. The few studies that have assessed the effect of nutritional interventions on body composition parameters regarding the Pro12Ala polymorphism did not demonstrate a significant association. A study with obese individuals who received a low-energy diet and were followed up for 1 year found no association between this polymorphism and changes in FFM and fat mass [15], while other weight loss intervention studies with overweight subjects also found no significant impact of this polymorphism on \%BF, FFM, and LM [36-39]. Individuals with a BMI between 25 and $40 \mathrm{~kg} / \mathrm{m}^{2}$ under a behavioural treatment programme for obesity based on a MedDiet did not exhibit any effect of the polymorphism on \%BF [40]. Our novel findings on the association of the Pro12Ala polymorphism with body composition parameters in 
Table 1 Sociodemographic, anthropometric, biochemical and polymorphism characteristics of the study groups

\begin{tabular}{|c|c|c|c|c|}
\hline Variable & $\begin{array}{l}\text { Total } \\
n=149\end{array}$ & $\begin{array}{l}\mathrm{OO} \\
\mathrm{n}=50\end{array}$ & $\begin{array}{l}\text { DieTBra } \\
n=49\end{array}$ & $\begin{array}{l}\text { DieTBra + OO } \\
n=50\end{array}$ \\
\hline Age & $39.63 \pm 8.82$ & $38.14 \pm 8.14$ & $39.14 \pm 8.16$ & $41.60 \pm 9.85$ \\
\hline \multicolumn{5}{|l|}{ Sex } \\
\hline Female & $127(85.2)$ & $45(90.0)$ & $40(81.6)$ & $42(84.0)$ \\
\hline Male & $22(14.8)$ & $5(10.0)$ & $9(18.4)$ & $8(16.0)$ \\
\hline Years of schooling & $9.60 \pm 3.05$ & $9.66 \pm 2.95$ & $9.86 \pm 2.96$ & $9.28 \pm 3.26$ \\
\hline \multicolumn{5}{|l|}{ Social status } \\
\hline$A / B$ & $34(22.8)$ & $13(26.0)$ & $10(20.4)$ & $11(22.0)$ \\
\hline C & $64(43.0)$ & $20(40.0)$ & $24(49.0)$ & $20(40.0)$ \\
\hline $\mathrm{D} / \mathrm{E}$ & $51(34.2)$ & $17(34.0)$ & $15(30.6)$ & 19 (38.0) \\
\hline Weight (kg) & $118.81 \pm 19.47$ & $117.38 \pm 18.69$ & $120.44 \pm 20.85$ & $118.64 \pm 19.10$ \\
\hline Height (m) & $1.60 \pm 0.07$ & $1.60 \pm 0.07$ & $1.61 \pm 0.08$ & $1.60 \pm 0.06$ \\
\hline $\mathrm{BMI}\left(\mathrm{kg} / \mathrm{m}^{2}\right)$ & $46.04 \pm 6.41$ & $45.77 \pm 6.27$ & $46.22 \pm 6.26$ & $46.13 \pm 6.79$ \\
\hline Fat mass $(\mathrm{kg})^{*}$ & $54.05 \pm 11.04$ & $52.39 \pm 11.77$ & $55.21 \pm 8.32$ & $54.37 \pm 11.03$ \\
\hline Fat free mass $(\mathrm{kg})^{*}$ & $53.42 \pm 8.04$ & $53.49 \pm 7.92$ & $54.16 \pm 7.42$ & $52.64 \pm 8.81$ \\
\hline Body fat percentage $(\%)^{*}$ & $51.62 \pm 5.18$ & $50.56 \pm 5.11$ & $51.54 \pm 4.70$ & $52.63 \pm 5.60$ \\
\hline Lean mass $(\mathrm{kg})^{*}$ & $51.07 \pm 7.93$ & $51.07 \pm 7.81$ & $51.84 \pm 7.5$ & $50.33 \pm 8.60$ \\
\hline Fasting glucose (mg/dL) & $109.95 \pm 45.24$ & $104.44 \pm 29.03$ & $107.43 \pm 35.15$ & $117.94 \pm 63.45$ \\
\hline Fasting insulin $(\mu \mathrm{U} / \mathrm{mL})$ & $23.42 \pm 14.86$ & $25.24 \pm 17.74$ & $24.40 \pm 14.64$ & $20.65 \pm 11.39$ \\
\hline HOMA-IR & $6.40 \pm 4.89$ & $6.78 \pm 5.87$ & $6.68 \pm 4.76$ & $5.75 \pm 3.87$ \\
\hline HbA1c (\%) & $6.30 \pm 1.43$ & $6.13 \pm 1.27$ & $6.26 \pm 1.42$ & $6.52 \pm 1.58$ \\
\hline Total cholesterol (mg/dL) & $189.12 \pm 38.10$ & $187.74 \pm 37.59$ & $184.31 \pm 33.40$ & $195.22 \pm 42.61$ \\
\hline HDL cholesterol (mg/dL) & $47.62 \pm 11.35$ & $47.18 \pm 10.32$ & $48.45 \pm 10.51$ & $47.26 \pm 13.16$ \\
\hline LDL cholesterol, (mg/dL) & $109.44 \pm 35.48$ & $106.94 \pm 29.96$ & $106.40 \pm 32.56$ & $114.76 \pm 42.51$ \\
\hline Triglyceride (mg/dL) & $160.31 \pm 78.40$ & $160.46 \pm 79.86$ & $154.76 \pm 87.76$ & $165.6 \pm 67.72$ \\
\hline MVPA (min/week) ${ }^{\dagger}$ & $44.29 \pm 61.11$ & $46.85 \pm 56.89$ & $49.10 \pm 69.57$ & $37.04 \pm 56.83$ \\
\hline Sedentary time $(\mathrm{min} / \mathrm{d})$ & $1176.85 \pm 83.28$ & $1171.35 \pm 73.25$ & $1171.24 \pm 81.74$ & $1187.85 \pm 8.12$ \\
\hline \multicolumn{5}{|l|}{ Pro12Ala polymorphism ${ }^{\ddagger}$} \\
\hline ProPro & $126(86.9)$ & $43(43.4)$ & $41(40.8)$ & $42(41.7)$ \\
\hline ProAla+AlaAla & $19(13.1)$ & $7(6.6)$ & $6(6.2)$ & $6(6.3)$ \\
\hline Pro alelle & $270(93.1)$ & $92(92.0)$ & $88(93.6)$ & $90(93.8)$ \\
\hline Ala alelle & $20(6.9)$ & $8(8.0)$ & $6(6.4)$ & $6(6.2)$ \\
\hline \multicolumn{5}{|l|}{$-174 G>C$ polymorphism ${ }^{\S}$} \\
\hline GG & $96(65.3)$ & 38 (39.6) & $29(30.2)$ & $29(30.2)$ \\
\hline$C G+C C$ & $51(34.7)$ & $12(23.5)$ & $18(35.3)$ & $21(41.2)$ \\
\hline $\mathrm{G}$ alelle & $238(81.0)$ & $87(87.0)$ & $75(79.8)$ & $76(76.0)$ \\
\hline$C$ alelle & $56(19.0)$ & 13 (13.0) & 19 (20.2) & $24(24.0)$ \\
\hline
\end{tabular}

There was no difference among the groups for any variable. Data are shown as absolute means \pm SD or N (\%). OO: olive oil group, DieTBra: traditional Brazilian diet group, DieTBra+OO: traditional Brazilian diet plus olive oil group, BMI: Body Mass Index, HOMA-IR: homeostatic model assessment for insulin resistance, HbA1C haemoglobin A1c, HDL high-density lipoprotein, $L D L$ low-density lipoprotein, MVPA moderate-to-vigorous physical activity

${ }^{*} n=111$, OO: $n=34$, DieTBra: $n=38$, DieTBra+OO: $n=39,{ }^{\dagger} n=140$, OO: $n=47$, BraDiet: $n=46$, BraDiet+OO: $n=47,{ }^{\ddagger} n=145,{ }^{\S} n=147$

severely obese individuals are important for the research field; however, larger sample sizes are necessary to confirm this result.

Anti-inflammatory mechanisms promoted by olive oil consumption might have had a role in the \%BF-, FFM-, and LM-related results of the current study. Obesity is considered a low-grade chronic inflammatory state [41]. Adipose tissue inflammation seems to have a dominant role in the development of sarcopenia, eventually leading to skeletal muscle inflammation and dysfunction [42]. 


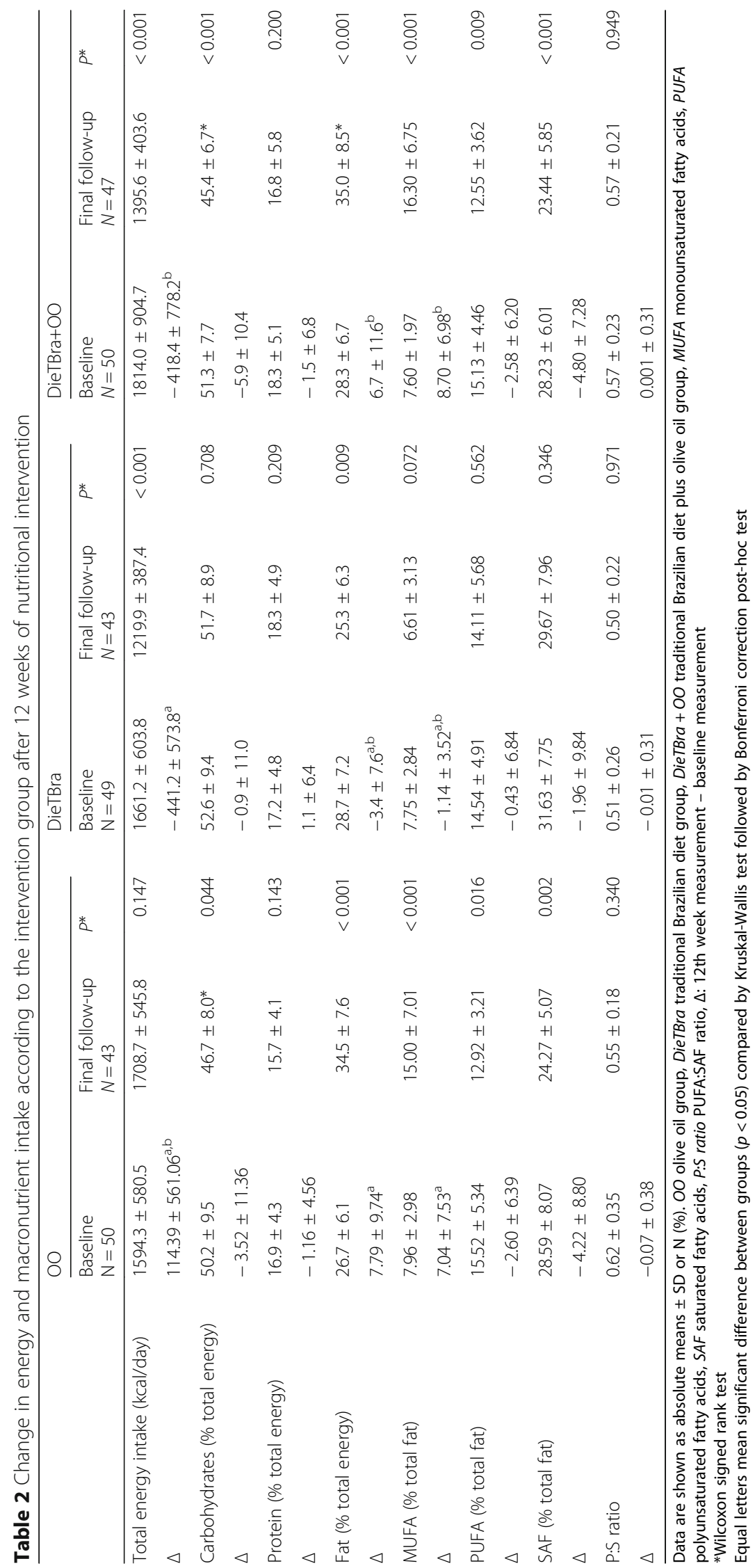




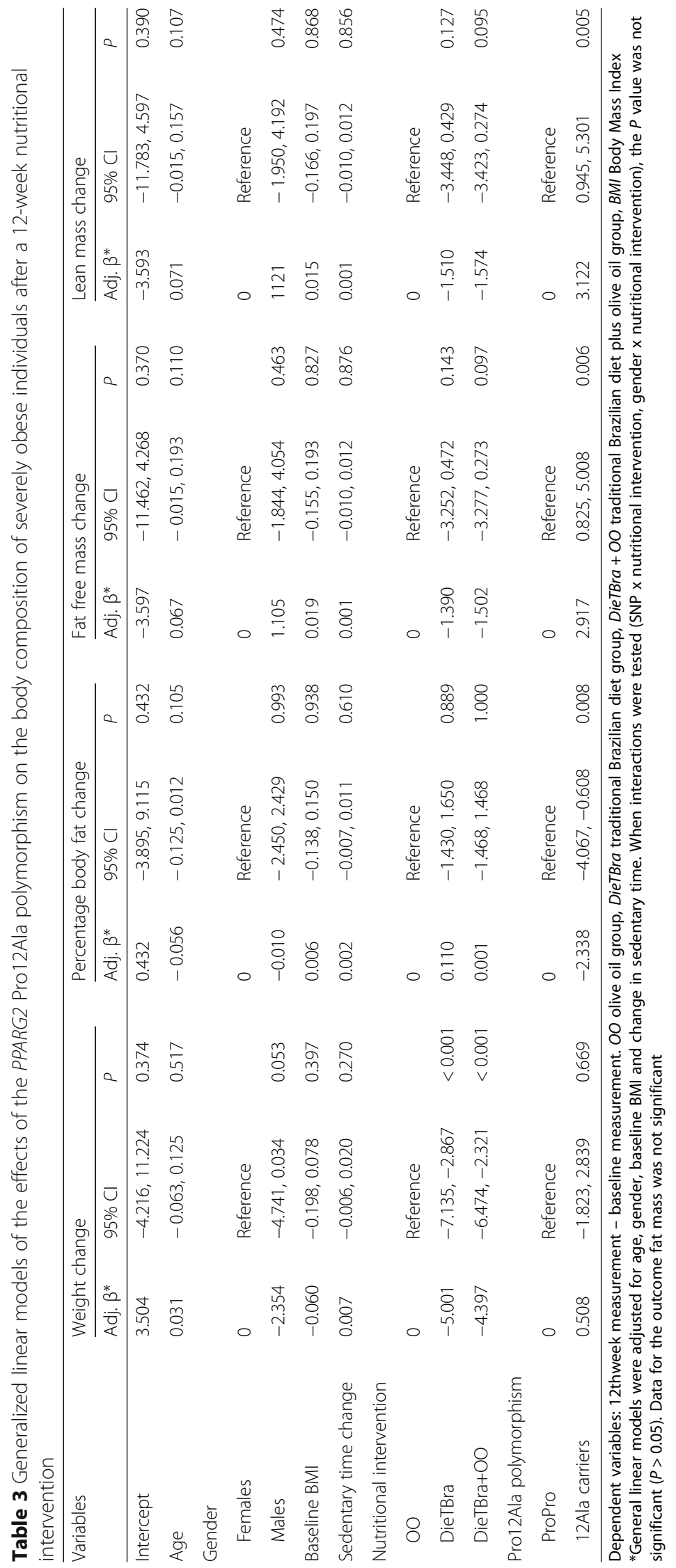


Table 4 Generalized linear models of the effects of the IL6 -174G/C polymorphism on the body composition of severely obese individuals after a 12-week nutritional intervention

\begin{tabular}{|c|c|c|c|c|c|c|}
\hline \multirow[t]{2}{*}{ Variables } & \multicolumn{3}{|c|}{ Weight change } & \multicolumn{3}{|c|}{ Fat mass change } \\
\hline & Adj. $\beta^{*}$ & $95 \% \mathrm{Cl}$ & $P$ & Adj. $\beta^{*}$ & $95 \% \mathrm{Cl}$ & $P$ \\
\hline Intercept & 3.969 & $-4.212,11.348$ & 0.369 & -0.754 & $-9.145,7.363$ & 0.860 \\
\hline Age & 0.026 & $-0.068,0.121$ & 0.585 & -0.027 & $-0.112,0.586$ & 0.541 \\
\hline \multicolumn{7}{|l|}{ Gender } \\
\hline Females & 0 & Reference & & Reference & & \\
\hline Males & -2.411 & $-4.752,-0.070$ & 0.044 & 1.281 & $-1.725,4.286$ & 0.404 \\
\hline Baseline BMI & -0.053 & $-0.188,0.081$ & 0.437 & 0.071 & $-0.110,0.253$ & 0.442 \\
\hline Sedentary time change & 0.008 & $-0.005,0.021$ & 0.235 & 0.003 & $-0.008,0.142$ & 0.588 \\
\hline \multicolumn{7}{|l|}{ Nutritional intervention } \\
\hline $\mathrm{OO}$ & 0 & Reference & & Reference & & \\
\hline DieTBra & -4.980 & $-7.103,-2.858$ & $<0.001$ & -1.495 & $-3.390,0.400$ & 0.122 \\
\hline DieTBra+OO & -4.406 & $-6.478,-2.335$ & $<0.001$ & -1.975 & $-3.798,-0.153$ & 0.034 \\
\hline \multicolumn{7}{|l|}{-174G > C polymorphism } \\
\hline GG & 0 & Reference & & Reference & & \\
\hline C carriers & -0.219 & $-1.956,1.519$ & 0.805 & -0.015 & $-1.513,1.484$ & 0.985 \\
\hline
\end{tabular}

Dependent variables: $12^{\text {th }}$ week measurement - baseline measurement. $O O$ olive oil group, DieTBra traditional Brazilian diet group, DieTBra $+O O$ traditional Brazilian diet plus olive oil group, BMI Body Mass Index

*General linear models were adjusted for age, gender, baseline BMI and change in sedentary time. When interactions were tested (SNP x nutritional intervention, gender $\mathrm{x}$ nutritional intervention), the $P$ value was not significant $(P>0.05)$. Data for the outcomes fat-free mass, body fat percentage and lean mass were not significant

Nutrigenomic studies with short-term and sustained olive oil consumption have shown the upregulation of genes related to an anti-inflammatory profile and the downregulation of genes related to inflammation [43-45]. We hypothesize that the improved anti-inflammatory profile promoted by the olive oil intake may have affected the cross-talk between fat and muscle, thus favouring the signalling pathways of skeletal muscle synthesis [42]. Additionally, even when weight loss was not significant, such as in the OO group, olive oil was able to promote favourable changes in the body composition of Ala carriers.

Another aspect regarding skeletal muscle inflammation and dysfunction refers to weight cycling. Obese individuals trying to lose weight usually experience periods of weight loss followed by periods of weight regain, leading to inadequate changes in FFM and LM, thus favouring the development of sarcopenia [46]. Therefore, it seems that weight loss intervention with olive oil is promising for improving body composition in Ala carriers. However, further investigation is needed to clarify the precise mechanisms underpinning the influence of olive oil on LM and FFM in severely obese individuals.

Interactions between diet and body composition changes related to the Pro12Ala polymorphism, such as those observed in the current study, have not been reported to date. Associations have been found in observational studies between a higher P:S ratio and MUFA intake and a lower BMI and \%BF [40, 47, 48]. Intervention studies have reported an interaction between a higher P:S ratio and a greater reduction in visceral adipose tissue [49], and between a higher total fat intake ( $\geq 42.6 \%$ of energy) and lower amount of weight loss [40]. Possibly, the interactions found in our study may be related not only to MUFA and PUFA but also to the antioxidant activity of phenolic compounds in olive oil, such as hydroxytyrosol and oleuropein; this possibility needs further investigation [45].

Our results have demonstrated that DieTBra and DieTBra+OO promoted a significant weight change that was independent of polymorphisms. Previous studies showed that a traditional Brazilian diet pattern was associated with a lower risk of overweight and obesity $[18-20,50]$. Evidence has suggested that olive oil consumption even outside the Mediterranean dietary pattern may improve health parameters [51], and olive oil consumption also promotes the higher consumption of vegetables, thereby contributing to a healthier eating pattern [52]. Olive oil consumption has been increasing among Brazilians [21], and as olive oil is easily incorporated into the diet, such consumption may be encouraged as part of a healthy dietary pattern.

The current study has several strengths, such as the use of an advanced technique, namely, DXA, to determine body composition, the objective measurement of 


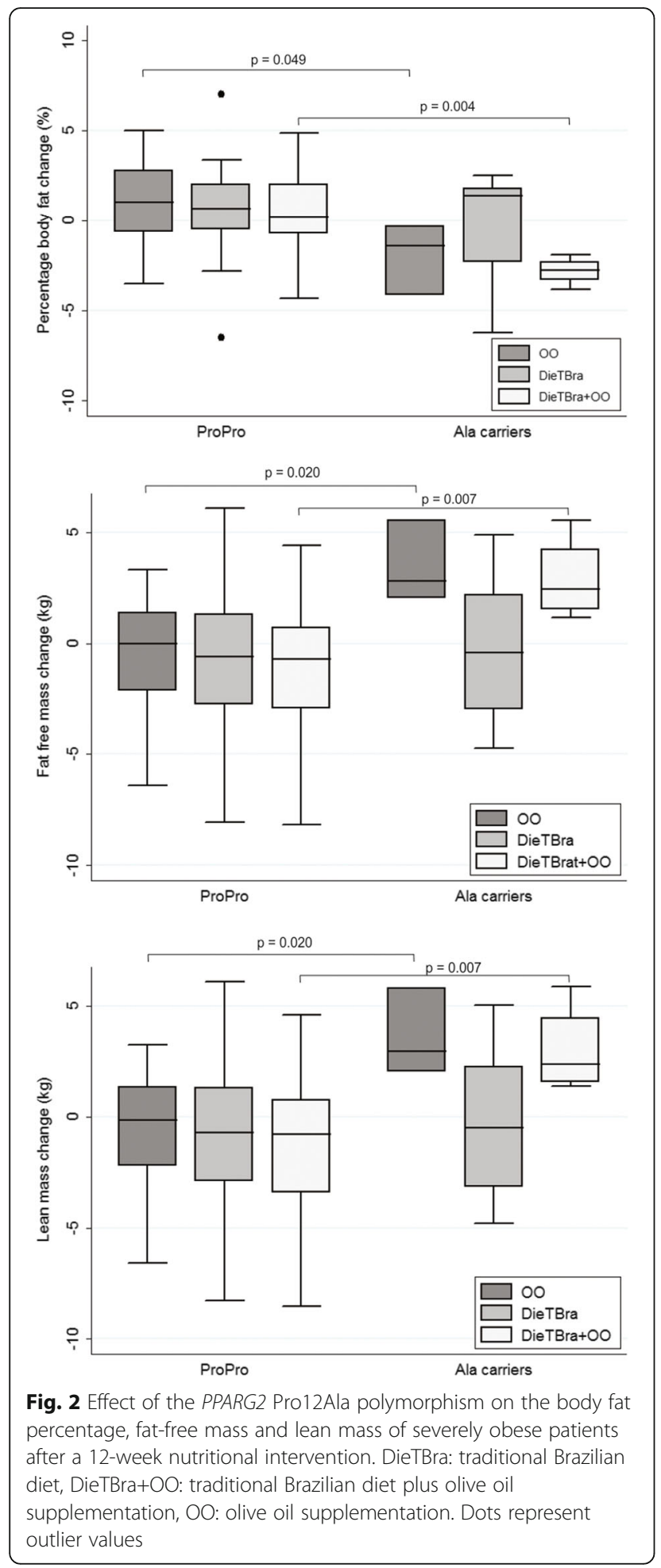

sedentary time and MVPA using a tri-axial accelerometer, the high retention rate, the real-world setting and intervention, and the randomized design. However, our study is not without limitations. These limitations include the sample size, especially the low number of individuals with the rare homozygote; the possibility of false-positive findings due to multiple comparisons; and the possibility of the under-reporting of food intake.

\section{Conclusions}

In summary, our findings highlight that extra virgin olive oil intake may modulate favourable body composition changes, thus decreasing \%BF and increasing LM and FFM in severely obese patients, even without weight loss, in the presence of the Ala allele of the Pro12Ala polymorphism. The beneficial effect of this gene-diet interaction may support the increasing body of evidence supporting the development of personalized interventions based on the genotype of the patient for more effective outcomes in the treatment of severe obesity.

\section{Additional file}

Additional file 1: Table S1. Changes in the body composition and metabolic parameters after 12 weeks of nutritional intervention. (DOCX 66 kb)

\section{Abbreviations}

\%BF: Percentage of body fat; ANOVA: Analysis of variance; BMI: Body mass index; DieTBra: Traditional Brazilian diet; DieTBra+OO: Traditional Brazilian diet plus extra virgin olive oil supplementation; DRI: Dietary reference intake; DXA: Dual-energy X-ray absorptiometry; FFM: Fat-free mass; GLM: General linear model; HbA1c: Haemoglobin A1c; HDL: High-density lipoprotein; HOMA-IR: Homeostasis model assessment of insulin resistance; LDL: Lowdensity lipoprotein; IL-6: Interleukin-6; LM: Lean mass;

MUFA: Monounsaturated fatty acids; MVPA: Moderate-to-vigorous physical activity; OO: Olive oil supplementation; P:S ratio: Polyunsaturated:saturated fatty acids ratio; PUFA: Polyunsaturated fatty acids; qPCR: quantitative polymerase chain reaction; SUS: Brazilian Unified Health System

\section{Acknowledgements}

The authors would like to thank the Clinical Research Unit of the Clinical Hospital/Federal University of Goiás and its technical staff for lending us the facilities to conduct this research and for providing technical support. The present work was partially funded by Fundação de Amparo à Pesquisa do Estado de Goiás (FAPEG) grant number 201310267000003. Furthermore, the authors are grateful to all persons who participated in the study.

\section{Funding}

This study was partially funded by the Goiás State Research Support Foundation (FAPEG) [grant number 201310267000003]. The funder had no role in the study design, data collection and analysis, decision to publish, or preparation of the manuscript.

\section{Availability of data and materials}

The datasets used and analysed during the current study are available from the corresponding author upon reasonable request.

\section{Authors' contributions}

APSR conceived the study; obtained funding; collected, analysed, and interpreted data; and wrote the manuscript. LPSR collected data and contributed to both the interpretation of data and the development of the introduction and discussion sections of the manuscript. EAS conceived the study, obtained funding, and coordinated the study. All authors participated in the design of the study protocols and in the revision and approval of the final manuscript. 


\section{Ethics approval and consent to participate}

Informed consent was obtained from all participants included in the study, and the study protocol was approved by the Research Ethics Committee of the Clinical Hospital/Federal University of Goiás (number 747.792).

\section{Consent for publication}

Not applicable.

\section{Competing interests}

The authors declare that they have no competing interests.

\section{Publisher's Note}

Springer Nature remains neutral with regard to jurisdictional claims in published maps and institutional affiliations.

Received: 2 May 2018 Accepted: 11 July 2018

Published online: 17 July 2018

\section{References}

1. Naukkarinen J, Rissanen A, Kaprio J, Pietiläinen KH. Causes and consequences of obesity: the contribution of recent twin studies. Int J Obes. 2012;36:1017-24.

2. Martínez JA, Milagro Fl. Genetics of weight loss: a basis for personalized obesity management. Trends Food Sci Technol. 2015;42:97-115.

3. El-Sayed Moustafa JS, Froguel P. From obesity genetics to the future of personalized obesity therapy. Nat Rev Endocrinol. 2013:9:402-13.

4. Marti A, Goyenechea E, Martínez JA. Nutrigenetics: a tool to provide personalized nutritional therapy to the obese. In: Simopoulos AP, Milner JA, editors. Personalized nutrition: translating Nutrigenetic/nutrigenomic research into dietary guidelines. Basel: Karger Publishers; 2010. p. 21-33.

5. Goni L, Cuervo M, Milagro I, Mart JA. Future perspectives of personalized weight loss interventions based on Nutrigenetic, epigenetic, and metagenomic data. Methods. 2016;149:2006.

6. Rudkowska I, Pérusse L. Individualized weight management: what can be learned from nutrigenomics and nutrigenetics? Prog Mol Biol Transl Sci. 2012;108:347-82.

7. Grygiel-Górniak B. Peroxisome proliferator-activated receptors and their ligands: nutritional and clinical implications - a review. Nutr J. 2014;13:1-10.

8. Mansoori A, Amini M, Kolahdooz F, Seyedrezazadeh E. Obesity and Pro12Ala polymorphism of peroxisome proliferator-activated receptor-gamma gene in healthy adults: a systematic review and meta-analysis. Ann Nutr Metab. 2015;67:104-18

9. Lapice E, Vaccaro O. Interaction between Pro12Ala polymorphism of PPARY2 and diet on adiposity phenotypes. Curr Atheroscler Rep. 2014;16:1-11.

10. Terry CF, Loukaci V, Green FR. Cooperative influence of genetic polymorphisms on interleukin 6 transcriptional regulation. J Biol Chem. 2000;275:18138-44.

11. Fishman D, Faulds G, Jeffery R, Mohamed-Ali V, Yudkin JS, Humphries S, et al. The effect of novel polymorphisms in the Interleukin-6 (IL-6) gene on IL6 transcription and plasma IL-6 levels, and an association with systemiconset juvenile chronic arthritis. J Clin Invest. 1998;102:1369-76.

12. Qi L, Zhang C, Van Dam RM, Hu FB. Interleukin-6 genetic variability and adiposity: associations in two prospective cohorts and systematic review in 26,944 individuals. J Clin Endocrinol Metab. 2007;92:3618-25.

13. Huth C, Illig T, Herder C, Gieger C, Grallert H, Vollmert C, et al. Joint analysis of individual participants' data from 17 studies on the association of the IL6 variant -174G $>C$ with circulating glucose levels, Interleukin-6 levels, and body-mass index. Ann Med. 2009;41:128-38.

14. Razquin C, Martinez JA, Martinez-Gonzalez MA, Fernández-Crehuet J, Santos $J M$, Marti A. A mediterranean diet rich in virgin olive oil may reverse the effects of the $-174 \mathrm{G} / \mathrm{C}$ IL6 gene variant on 3-year body weight change. Mol Nutr Food Res. 2010;54:75-82.

15. Goyenechea E, Parra MD, Martínez JA. Weight regain after slimming induced by an energy-restricted diet depends on interleukin- 6 and peroxisome-proliferator-activated-receptor-gamma2 gene polymorphisms. Br J Nutr. 2006;96:965-72.

16. Sesti G, Perego L, Cardellini M, Andreozzi F, Ricasoli C, Vedani P, et al. Impact of common polymorphisms in candidate genes for insulin resistance and obesity on weight loss of morbidly obese subjects after laparoscopic adjustable gastric banding and hypocaloric diet. J Clin Endocrinol Metab. 2005;90:5064-9.
17. Martínez-González MA, Salas-Salvadó J, Estruch R, Corella D, Fitó M, Ros E. Benefits of the Mediterranean diet: insights from the PREDIMED study. Prog Cardiovasc Dis. 2015;58:50-60.

18. Cunha DB, de Almeida RMVR, Sichieri R, Pereira RA. Association of dietary patterns with $\mathrm{BMI}$ and waist circumference in a low-income neighbourhood in Brazil. Br J Nutr. 2010;104:908-13.

19. Sichieri R. Dietary patterns and their associations with obesity in the Brazilian City of Rio de Janeiro. Obes Res. 2002;10:42-8.

20. Sichieri R, Castro JFG, Moura AS. Factors associated with dietary patterns in the urban Brazilian population. Cad Saude Publica. 2003;19:47-53.

21. International Olive Council. Market Newsletter Nº 91 - November 2015. 2015;

22. Satija A, Yu E, Willett WC, Hu FB. Understanding nutritional epidemiology and its role in policy. Adv Nutr An Int Rev J. 2015;6:5-18.

23. Monteiro CA, Cannon G. The impact of transnational "big food" companies on the south: a view from Brazil. PLoS Med. 2012:9

24. Brasil. Guia alimentar para a população brasileira. 2nd ed. Brasília: Ministério Da Saúde, Secretaria de Atenção à Saúde, Departamento de Atenção Básica; 2014.

25. Horie LM, Gonzalez MC, Torrinhas RS, Cecconello I, Waitzberg DL. New specific equation to estimate resting energy expenditure in severely obese patients. Obesity (Silver Spring). 2011;19:1090-4.

26. Institute of Medicine. Dietary reference intakes for energy, carbohydrate, Fiber, fat, fatty acids, cholesterol, protein, and amino acids (macronutrients). Washington: The National Academies Press; 2005.

27. Hill JO, Wyatt HR, Peters JC. Energy balance and obesity. Circulation. 2012; 126:126-32.

28. World Health Organization. Global recommendations on physical activity for health. World Heal Organ. 2010;

29. Lohman TG, Roche AF, Martorell R. Anthropometric standardization reference manual. Champaing: Human Kinetics Books; 1988.

30. Di Cesare M, Bentham J, Stevens GA, Zhou B, Danaei G, Lu Y, et al. Trends in adult body-mass index in 200 countries from 1975 to 2014: a pooled analysis of 1698 population-based measurement studies with 19.2 million participants. Lancet. 2016:387:1377-96.

31. IAEA. Library cataloguing in publication data. Dual energy $X$ ray absorptiometry for bone mineral density and body composition assessment. Vienna: International Atomic Energy Agency; 2010.

32. Moshfegh AJ, Rhodes DG, Baer DJ, Murayi T, Clemens JC, Rumpler WV, et al. The US Department of Agriculture Automated Multiple-Pass Method reduces bias in the collection of energy intakes. Am J Clin Nutr. 2008;88:324-32

33. Hildebrand $M$, Van Hees VT, Hansen BH, Ekelund U. Age group comparability of raw accelerometer output from wrist-and hip-worn monitors. Med Sci Sports Exerc. 2014;46:1816-24.

34. Silva ICM, van Hees VT, Ramires W, Knuth AG, Bielemann RM, Ekelund U, et al. Physical activity levels in three Brazilian birth cohorts as assessed with raw triaxial wrist accelerometry. Int J Epidemiol. 2014;43:1959-68.

35. Matthews DR, Hosker JP, Rudenski AS, Naylor BA, Treacher DF, Turner RC. Homeostasis model assessment: insulin resistance and beta-cell function from fasting plasma glucose and insulin concentrations in man. Diabetologia. 1985;28:412-9.

36. Nicklas BJ, van Rossum EFC, Berman DM, Ryan AS, Dennis KE, Shuldiner AR. Genetic variation in the peroxisome subsequent weight regain. Diabetes. 2001;50:2172-6.

37. Kim KS, Choi SM, Shin SU, Yang HS, Yoon Y. Effects of peroxisome proliferator-activated receptor- $\gamma 2$ Pro12Ala polymorphism on body fat distribution in female Korean subjects. Metabolism. 2004;53:1538-43.

38. Vogels N, Mariman ECM, Bouwman FG, Kester ADM, Diepvens K, Westerterp-plantenga MS. Relation of weight maintenance and dietary restraint to peroxisome proliferator - activated receptor $\gamma 2$, glucocorticoid receptor, and ciliary neurotrophic factor polymorphisms. Am J Clin Nutr. 2005;82:740-6.

39. Matsuo $T$, Nakata $Y$, Katayama $Y$, lemitsu M, Maeda $S$, Okura T, et al. PPARG genotype accounts for part of individual variation in body weight reduction in response to calorie restriction. Obesity (Silver Spring). 2009;17:1924-31.

40. Garaulet M, Smith CE, Hernandez-Gonzalez T, Lee Y, Ordovas JM. PPARY Pro12Ala interacts with fat intake for obesity and weight loss in a behavioural treatment based on the Mediterranean diet. Mol Nutr Food Res. 2011;55:1771-9.

41. de Heredia FP, Gómez-Martínez S, Marcos A. Obesity, inflammation and the immune system. Proc Nutr Soc. 2012;71:332-8. 
42. Kalinkovich A, Livshits G. Sarcopenic obesity or obese sarcopenia: a cross talk between age-associated adipose tissue and skeletal muscle inflammation as a main mechanism of the pathogenesis. Ageing Res Rev. 2017:35:200-21.

43. van Dijk SJ, Feskens EJM, Bos MB, Hoelen DWM, Heijligenberg R, Bromhaar $M G$, et al. A saturated fatty acid - rich diet induces an obesity-linked proinflammatory gene expression profile in adipose tissue of subjects at risk of metabolic syndrome. Am J Clin Nutr. 2009;90:1656-64.

44. Konstantinidou V, Khymenets O, Fito M, De La Torre R, Anglada R, Dopazo $A$, et al. Characterization of human gene expression changes after olive oil ingestion: an exploratory approach. Folia Biol (Praha). 2009;91:85-91.

45. Piroddi M, Albini A, Fabiani R, Giovannelli L, Luceri C, Natella F, et al. Nutrigenomics of extra-virgin olive oil: a review. Biofactors. 2016:43:17-41.

46. Santarpia L, Contaldo F, Pasanisi F. Body composition changes after weightloss interventions for overweight and obesity. Clin Nutr. 2013;32:157-61.

47. Luan J, Browne PO, Harding A, Halsall DJ, O'Rahilly S, Krishna Chatterjee VK, et al. Evidence for gene-nutrient interaction at the PPARY locus. Diabetes. 2000;50:686-9.

48. Memisoglu A, Hu FB, Hankinson SE, Manson JE, De Vivo I, Willett WC, et al. Interaction between a peroxisome proliferator-activated receptor $\gamma$ gene polymorphism and dietary fat intake in relation to body mass. Hum Mol Genet. 2003:12:2923-9.

49. Franks PW, Jablonski KA, Delahanty L, Hanson RL, Kahn SE, Altshuler D, et al. The Pro12Ala variant at the peroxisome proliferator-activated receptor $\gamma$ gene and change in obesity-related traits in the diabetes prevention program. Diabetologia. 2007:50:2451-60.

50. Pinheiro S, Arruda M, Augusto A, Kac G, Amélia A, Vilela F, et al. Dietary patterns are associated with excess weight and abdominal obesity in a cohort of young Brazilian adults. Eur J Nutr. 2015;55:2081-91.

51. López-Miranda J, Pérez-Jiménez F, Ros E, De Caterina R, Badimón L, Covas $\mathrm{Ml}$, et al. Olive oil and health: summary of the II international conference on olive oil and health consensus report, Jaén and Córdoba (Spain) 2008. Nutr Metab Cardiovasc Dis. 2010;20:284-94

52. Panagiotakos DB, Pitsavos C, Stefanadis C. Dietary patterns: a Mediterranean diet score and its relation to clinical and biological markers of cardiovascular disease risk. Nutr Metab Cardiovasc Dis. 2006;16:559-68.

Ready to submit your research? Choose BMC and benefit from:

- fast, convenient online submission

- thorough peer review by experienced researchers in your field

- rapid publication on acceptance

- support for research data, including large and complex data types

- gold Open Access which fosters wider collaboration and increased citations

- maximum visibility for your research: over $100 \mathrm{M}$ website views per year

At $\mathrm{BMC}$, research is always in progress.

Learn more biomedcentral.com/submissions 